\title{
Investigation of the SV40 - human cancer association: Look for the full signature of the virus
}

\author{
Keerti V. Shah ${ }^{\mathrm{a}, *}$ and Dana E.M. Rollison ${ }^{\mathrm{b}}$ \\ ${ }^{a}$ Department of Molecular Microbiology and \\ Immunology, and \\ ${ }^{\mathrm{b}}$ Department of Epidemiology, Johns Hopkins \\ University, School of Public Health, 615 North Wolfe \\ Street, Baltimore, MD 21205, USA
}

In the controversy about the association of simian virus 40 with human cancers, the greatest problem is the ascertainment of SV40 exposure. This difficulty would be resolved if one were to look for all components of SV40 infection. How does SV 40 circulate in the human community? Do cancer patients with SV40-positive tumors have serological correlates of SV 40 infection and of SV40-induced cancer? SV40 association with a cancer should be studied in the context of the known risk factors for that cancer. The tumor cell-virus relationship should be characterized with respect to viral integration and viral localization to the tumor cell. Specimens should be masked and the assays should include panels of specimens to estimate analytic sensitivity and specificity. In view of the rarity of some of the tumors reported to be associated with SV40, a multi-institutional investigation initiated and coordinated by the NIH would be most effective.

Keywords: SV40, human cancers

\section{Introduction and background}

Since the early 1990's, several teams of investigators have addressed the controversial topic of the relationship of SV40 to human cancers. The data from a large number of published reports are conflicting in a very fundamental way and cannot be reconciled. SV40 has been reported to be recovered from cancers of many histologic types and at several sites and from cancers of adults and children. Of these cancers, malignant pleu-

*Address for correspondence: Tel.: +1 410955 3189; Fax: +1 410955 0105; E-mail: kvshah@jhsph.edu. ral mesothelioma and pediatric brain tumors have been studied most extensively. Our remarks are addressed primarily to studies of these tumors.

The evidence in favor of an association of SV40 with these tumors (reviewed in $[1,2]$ ) may be summarized as follows. SV40 sequences have been recovered from these tumors by many different laboratories, and infectious SV40 has been recovered occasionally. There is sequence variation among the isolates suggesting that the positive results are not due to contamination from a common source. SV40 regulatory region in the isolates from the cancer tissues has a single 72 bp element, similar to primary isolates of SV40. In addition, SV40 transcripts, SV40 T antigen and p53-SV40 T antigen complexes have been reported to be present in some tumors. SV40 has been identified in tumor cell lines and administration of SV40 anti-sense sequences has been reported to reverse the transformed phenotype of the tumor cells. The human-derived pleural mesothelium has been shown to be more readily transformable by SV40 than human fibroblasts. The evidence against this association (reviewed in $[3,4]$ ) consists of a lack of reproducibility of the positive results, absence of tumorspecific or human-specific sequences in the SV40 sequences derived from the human cancers and the failure to find a plausible explanation of how the patients with SV40-positive tumors became infected with SV40. The interpretations of the data have ranged from the idea that the virus contributes to the development of some human cancers and that SV40-based immunotherapy may have a place in the prevention and treatment of specific cancers to skepticism about even the presence of the virus in human tissues.

\section{Suggestions for future research}

We make the following suggestions that might help in resolving the controversy. 


\subsection{Define how SV40 circulates in human communities}

Many of the reports that describe SV40 in human cancers imply that SV40, a macaque polyomavirus which had contaminated some lots of polio vaccine and for which there is no known animal host in the USA and Europe, is now adapted to humans and is circulating freely in human communities. At present, there is little evidence to support this claim. In the absence of knowledge of the epidemiology of SV40 transmission in humans, it is difficult to interpret the data from cancer patients.

\subsection{Focus on patients with cancer rather than on archived cancer tissues. Look for the full signature of SV40}

Almost all of the investigations, to date, have studied archived tissues. This has severely restricted the investigators' ability to fully ascertain SV40 exposure. If the focus of the studies were shifted to the living patient, additional measures of exposure to SV40 (other than amplification of SV40 sequences from tumor tissues) would become available, such as antibodies to SV40 capsids as evidence of past infection, antibodies to SV40 T protein as suggestive of a role of SV40 in tumor development, and urinary shedding of the virus as evidence of chronic SV40 infection.

Focusing on the living patient would help resolve the question of mode of transmission of the virus in pediatric brain tumors. It has been proposed that in patients with SV40-positive pediatric brain tumors the virus may have been transmitted intra-uterine from an infected mother to the fetus. If this were the case, the mother of the patient would be expected to have had significant SV40 viremia during pregnancy and therefore a strong antibody response to the virus. Evidence of SV40 infection in mothers of patients with SV40positive brain tumors, but not in mothers of patients with SV40-negative brain tumors, would provide compelling evidence for the proposed mode of transmission.

\subsection{Examine SV40 exposure in the context of other known risk factors for the cancer}

Asbestos exposure is a well-documented and universally accepted risk factor for malignant mesothelioma. Studies of the SV40-mesothelioma association, which do not take into account the asbestos exposure of the same subjects, will be difficult to interpret with respect to the contribution of SV40 in the development of the disease; i.e., whether SV40 is truly a risk factor, and if it is, whether its effects are independent of the effect of asbestos. It would be worthwhile to estimate the extent of SV40 exposure in individuals who are exposed to asbestos but are at present disease-free and to include studies for asbestos fibers in mesothelioma specimens that are investigated for SV40 sequences.

\subsection{Characterize fully the tumor cell-SV4O relationship}

It is not clear if, in the SV40-positive tumors, the virus is present in every tumor cell and if its genome is integrated into the cellular DNA. Studies addressing this question have been difficult to carry out because the virus in the tumors is reported to be present in very low copy numbers. Elucidation of the nature of the cell-virus relationship in SV40-positive tumors is crucial for interpretation of the role of the virus in tumor development. Detailed studies of even a few specimens can provide critical insights; for example, analyses of a single human papillomavirus (HPV)-16-positive oral cancer and of the cell line established from that cancer provided persuasive evidence that the tumor was probably etiologically linked to HPV-16 infection [5].

\subsection{Apply rigorous and transparent standards for laboratory assays}

This can be achieved by: (a) masking and randomordering of all specimens including positive and negative controls, (b) inclusion, in each run, of titrations of viral DNA to estimate analytical sensitivity and of negative controls to assure specificity, (c) tests of duplicate aliquots of the tissue DNAs, on different days, to assess the reproducibility of the results, and (d) testing the specimens in more than one laboratory. Relatively few studies have employed such standards.

\subsection{The NIH should spearhead these efforts}

Pediatric brain tumors and mesotheliomas are rare and patients with these tumors do not survive for long. A multi-institutional study, with a single protocol, of patients with these tumors can be carried out most efficiently if the NIH were to initiate and coordinate this effort. 


\section{References}

[1] J.S. Butel and J.A. Lednicky, Cell and molecular biology of simian virus 40: Implications for human infection and disease (review), JNCI 91 (1999), 119-134.

[2] M. Carbone, P. Rizzo and H. Pass, Simian virus 40: the link with human malignant mesothelioma is well established, Anticancer Res 20 (1999), 875-878.

[3] D.E.M. Rollison and K.V. Shah, The epidemiology of SV40 infection due to contaminated polio vaccines: Relation of the virus to human cancer, in: The Human Polyomaviruses JC, BK and SV40: Molecular and Clinical Perspectives, K. Khalili and G.L. Stoner, eds, John Wiley \& Sons, Inc., New York, in press.

[4] K.V. Shah, Does SV40 infection contribute to the development of human cancers? Rev Med Virol 10 (2000), 31-43.

[5] R.D.M. Steenbergen, A.J.A. Hermsen, J.M.M. Walboomers, H. Jenje, F. Arwert, C.J.L.M. Meijer and P.J.F. Snijders, Integrated human papillomavirus type 16 and loss of heterozygosity at $11 \mathrm{a} 22$ and $18 \mathrm{q} 21$ in an oral carcinoma and its derivative cell line, Cancer Res 55 (1995), 5465-5471. 


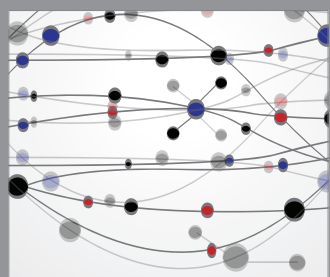

The Scientific World Journal
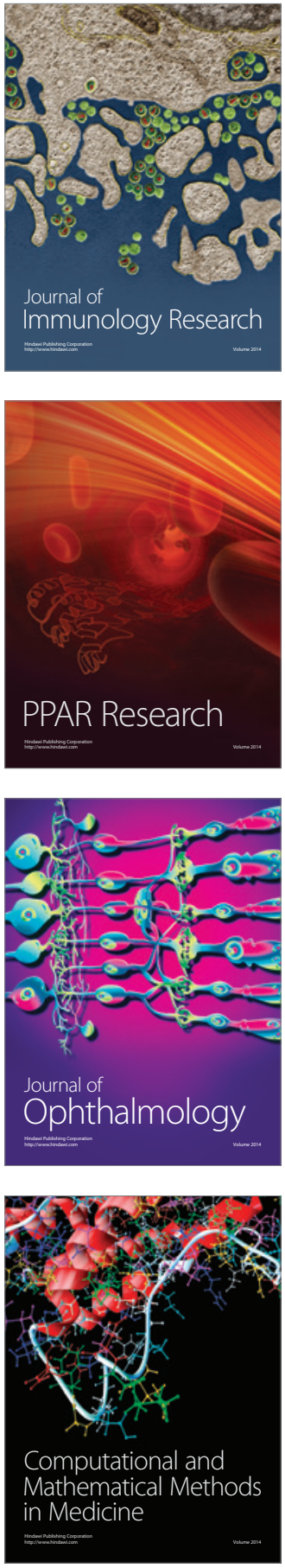

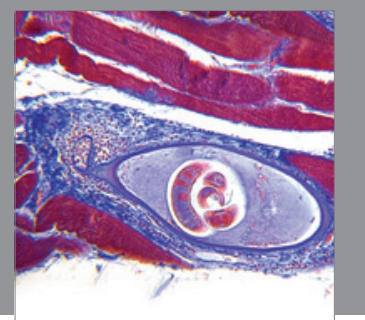

Gastroenterology

Research and Practice
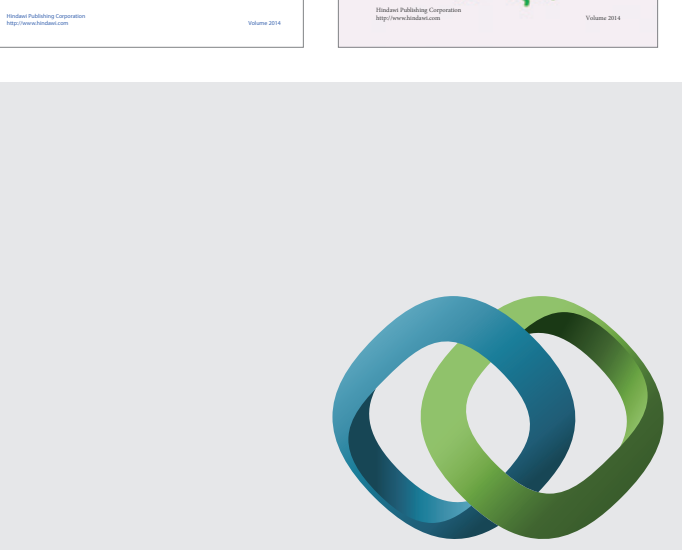

\section{Hindawi}

Submit your manuscripts at

http://www.hindawi.com
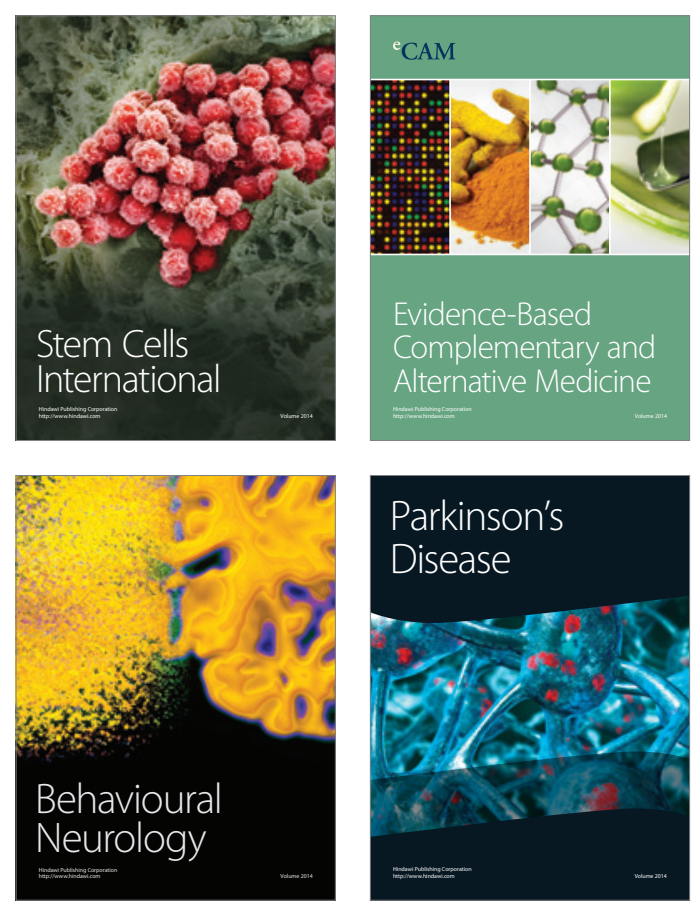

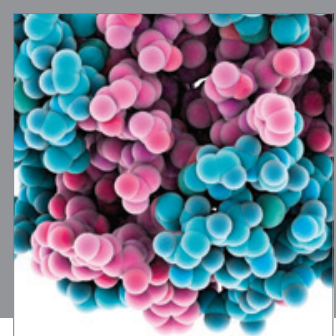

Journal of
Diabetes Research

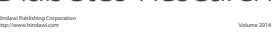

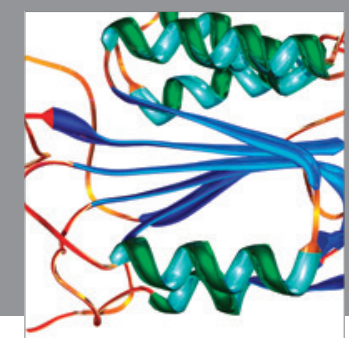

Disease Markers
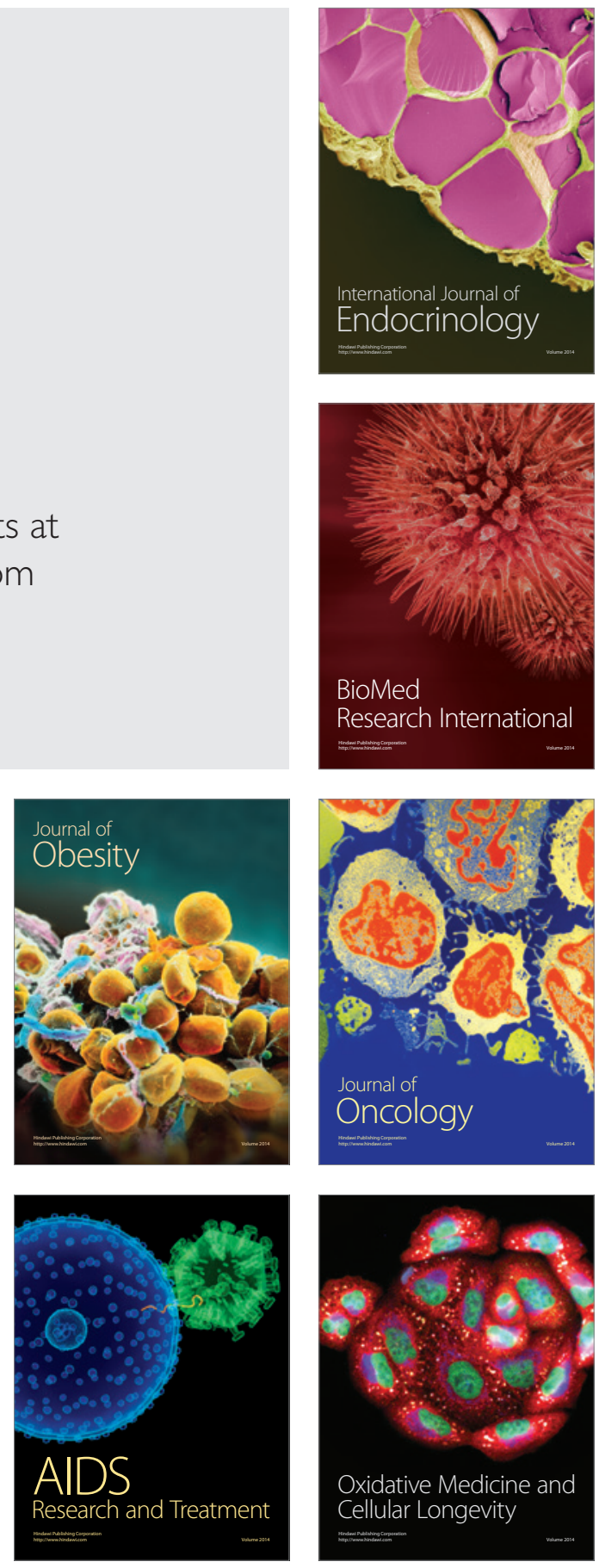